\title{
Quantum oscillations in underdoped $\mathrm{YBa}_{2} \mathrm{Cu}_{3} \mathrm{O}_{6.5}$
}

\author{
Cyril Jaudet, ${ }^{1}$ Julien Levallois, ${ }^{1}$ Alain Audouard,${ }^{1}$ David Vignolles, ${ }^{1}$ Baptiste Vignolle, ${ }^{1}$ \\ Ruixing Liang, ${ }^{2,3}$ D.A. Bonn, ${ }^{2,3}$ W.N. Hardy, ${ }^{2,3}$ N.E. Hussey, ${ }^{4}$ Louis Taillefer, ${ }^{3,5}$ and Cyril Proust ${ }^{1,3, *}$ \\ ${ }^{1}$ Laboratoire National des Champs Magnétiques Intenses (CNRS), Toulouse, France \\ ${ }^{2}$ Department of Physics and Astronomy, University of British Columbia, Vancouver, Canada \\ ${ }^{3}$ Canadian Institute for Advanced Research, Toronto, Canada \\ ${ }^{4}$ H. H. Wills Physics Laboratory, University of Bristol, Bristol, U.K. \\ ${ }^{5}$ Département de physique \& $R Q M P$, Université de Sherbrooke, Sherbrooke, Canada
}

\begin{abstract}
Shubnikov-de Haas and de Haas-van Alphen effects have been measured in the underdoped high temperature superconductor $\mathrm{YBa}_{2} \mathrm{Cu}_{3} \mathrm{O}_{6.51}$. Data are in agreement with the standard LifshitzKosevitch theory, which confirms the presence of a coherent Fermi surface in the ground state of underdoped cuprates. A low frequency $F=530 \pm 10 \mathrm{~T}$ is reported in both measurements, pointing to small Fermi pocket, which corresponds to $2 \%$ of the first Brillouin zone area only. This low value is in sharp contrast with that of overdoped $\mathrm{Tl}_{2} \mathrm{Ba}_{2} \mathrm{CuO}_{6+\delta}$, where a high frequency $F=18 \mathrm{kT}$ has been recently reported and corresponds to a large hole cylinder in agreement with band structure calculations. These results point to a radical change in the topology of the Fermi surface on opposing sides of the cuprate phase diagram.
\end{abstract}

PACS numbers: 74.25.Jb, 74.25.Bt, 74.25.Ha, 74.72.Bk

The first unambiguous observation of quantum oscillations (QO) in underdoped $\mathrm{YBa}_{2} \mathrm{Cu}_{3} \mathrm{O}_{6.5}$ [1] has created a lot of interest concerning the exact nature and the origin of the Fermi surface (FS) in the pseudogap phase of cuprates. If $\mathrm{QO}$ are interpreted as the usual consequence of the quantization of closed orbits in a magnetic field, they show that the FS consists of small pockets. It contrasts with angle-resolved photoemission spectroscopy (ARPES) which shows an apparent destruction of the FS producing a set of disconnected Fermi arcs [2]. The apparent contradiction between the two sets of measurements is not yet resolved [3 5$]$. Shubnikov-de Haas effect has been confirmed in the stoichiometric compound $\mathrm{YBa}_{2} \mathrm{Cu}_{4} \mathrm{O}_{8}$ [6, 7], indicating that $\mathrm{QO}$ are generic to the $\mathrm{CuO}_{2}$ planes rather than some feature of the band structure specific to $\mathrm{YBa}_{2} \mathrm{Cu}_{3} \mathrm{O}_{6.5}[\underline{6}, 8]$. More recently, de Haas-van Alphen effect (dHvA) has been reported in underdoped $\left.\mathrm{YBa}_{2} \mathrm{Cu}_{3} \mathrm{O}_{6.5}, 9,10\right]$, which confirmed the existence of a well-defined, closed, and coherent, FS via a thermodynamic probe.

In this paper we report a set of new data of Shubnikovde Haas $(\mathrm{SdH})$ in an underdoped cuprate $\mathrm{YBa}_{2} \mathrm{Cu}_{3} \mathrm{O}_{6.51}$ obtained both in the in-plane and Hall resistances. We compare the results with measurements of the $\mathrm{dHvA}$ at the same doping level published in ref. [9].

Transverse magnetoresistance $\left(R_{x x}\right)$ and Hall resistance $\left(R_{x y}\right)$ were measured down to $1.5 \mathrm{~K}$ in pulsed magnetic fields up to $60 \mathrm{~T}$. Current and field were applied along the $\mathbf{a}$ axis and normal to the $\mathrm{CuO}$ planes, respectively. A commercial piezoresistive microcantilever [11] was used for torque measurements in a dilution fridge down to $0.5 \mathrm{~K}$ in pulsed magnetic fields up to $59 \mathrm{~T}$. The crystal was glued to the beam of the cantilever with an angle of approximately $\theta \sim 5^{\circ}$ between the direction of the magnetic field and the normal to the $\mathrm{CuO}_{2}$ planes
[9].

We used two detwinned single crystals of $\mathrm{YBa}_{2} \mathrm{Cu}_{3} \mathrm{O}_{6.51}$ flux-grown in a non-reactive $\mathrm{BaZrO}_{3}$ crucible [12]. The dopant oxygen atoms are ordered into an ortho-II superstructure of alternating full and empty $\mathrm{CuO}$ chains which corresponds to a superconducting temperature of $T_{c}=57.5 \mathrm{~K}$ and therefore a doping of about $p \sim 0.1[13$.

Fig. 1(a) shows raw data of torque (defined as $\tau=$ $|\vec{M} \times \vec{B}|$ where $M$ is the magnetization) in one sample of $\mathrm{YBa}_{2} \mathrm{Cu}_{3} \mathrm{O}_{6.51}$ at different temperatures. The torque signal displays clear dHvA oscillations in high magnetic fields. Above $30 \mathrm{~T}$, the torque varies almost linearly with magnetic field which allow us to subtract a monotonic background from the data in order to derive the oscillatory part. Eight oscillations can be resolved at $T=0.5$ K.

Fig. 1(b) and Fig. 1(c) show raw data of the Hall resistance and of the transverse magnetoresistance, respectively, in another sample of $\mathrm{YBa}_{2} \mathrm{Cu}_{3} \mathrm{O}_{6.51}$ at different temperatures. Oscillatory parts of $R_{x y}$ and $R_{x x}$ are obtained after substraction of a polynomial background.

The oscillatory part of the resistance $(\Delta R)$ and of the magnetization $(\Delta M)$ are analyzed in the framework of the standard Lifshitz-Kosevich (LK) theory for a twodimensional generalized Fermi liquid [14]:

$$
\Delta R, \Delta M \propto R_{T} R_{D} \cos \left[2 \pi\left(\frac{F}{B}-\gamma\right)\right]
$$

where $F$ is the oscillation frequency and $\gamma$ a phase factor. The amplitude is given by $A \propto R_{T} R_{D}$, where $R_{T}=$ $\alpha T m^{*} / B \sinh \left[\alpha T m^{*} / B\right]$ and $R_{D}=\exp \left[-\alpha T_{D} m^{*} / B\right]$ are the thermal and Dingle damping factors, respectively. $m^{*}$ is the cyclotron effective mass, $T_{D}$ is the Dingle tem- 


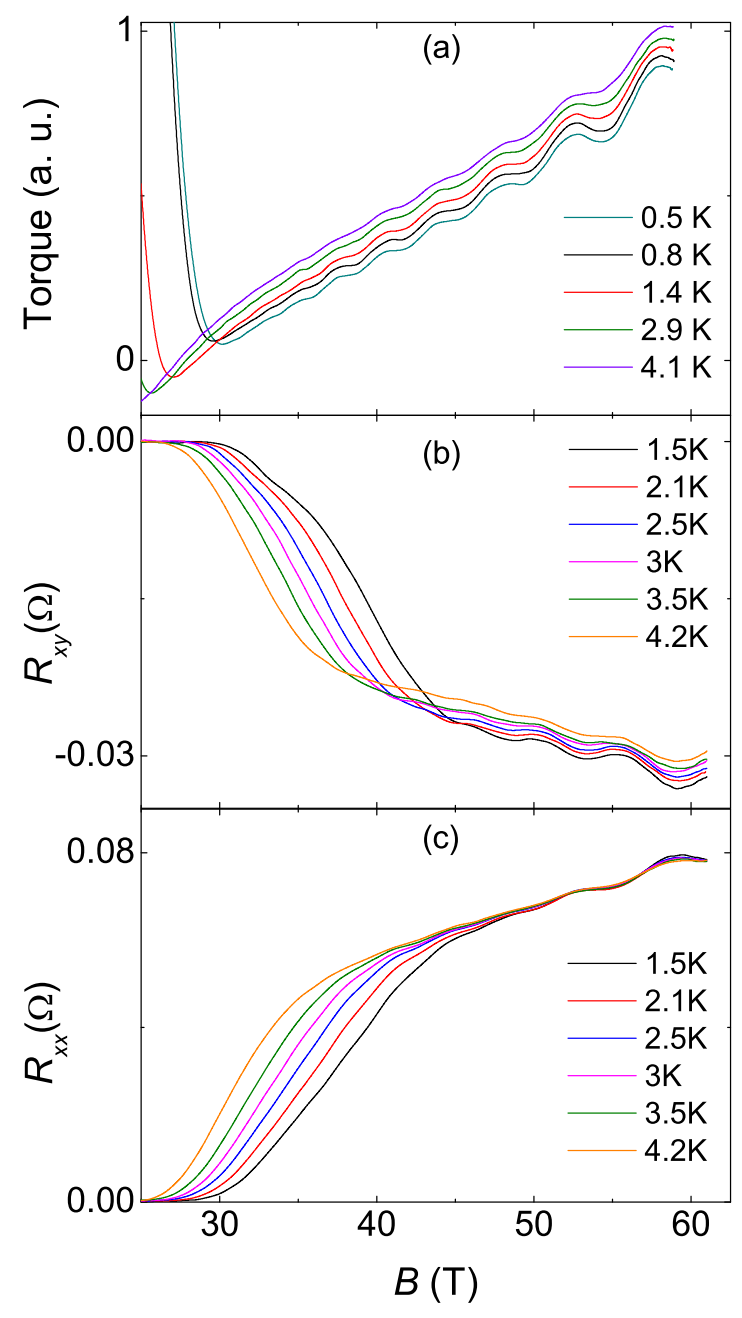

FIG. 1: Field dependence of the torque (a), Hall resistance (b) and magnetoresistance (c) at different temperatures. The curves of the torque are shifted for clarity.

perature $\left(T_{D} \propto \tau^{-1}\right)$ and $\alpha=2 \pi^{2} k_{B} m_{0} / e \hbar(\sim 14.69$ $\mathrm{T} / \mathrm{K})$.

Fourier analysis of the oscillatory part of the QO all give a single peak at about $F=530 \pm 10 \mathrm{~T}$, which is also consistent with the previous study [1]. The amplitude of the oscillations in $R_{x x}$ is large enough in the present study to deduce reliable parameters from the Fourier analysis. In particular, it is clear from Fig. 1 that the phase shift between the transverse magnetoresistance $R_{x x}$ and the Hall resistance $R_{x y}$ is about $\pi$, as expected in the case of an electron pocket. Such a pocket in the FS of underdoped $\mathrm{YBa}_{2} \mathrm{Cu}_{3} \mathrm{O}_{y}$ has been inferred from Hall effect measurements [15] and confirmed in the present study in a different sample.

Fig. 2 displays the temperature dependence of the oscillation amplitudes. Good agreement with the LK theory is observed, giving an effective mass $m^{*}=1.8 \pm$ $0.1 m_{0}$. The inset of Fig. 2 shows the effective mass deduced from $\mathrm{dHvA}$ and $\mathrm{SdH}$ in different field windows.

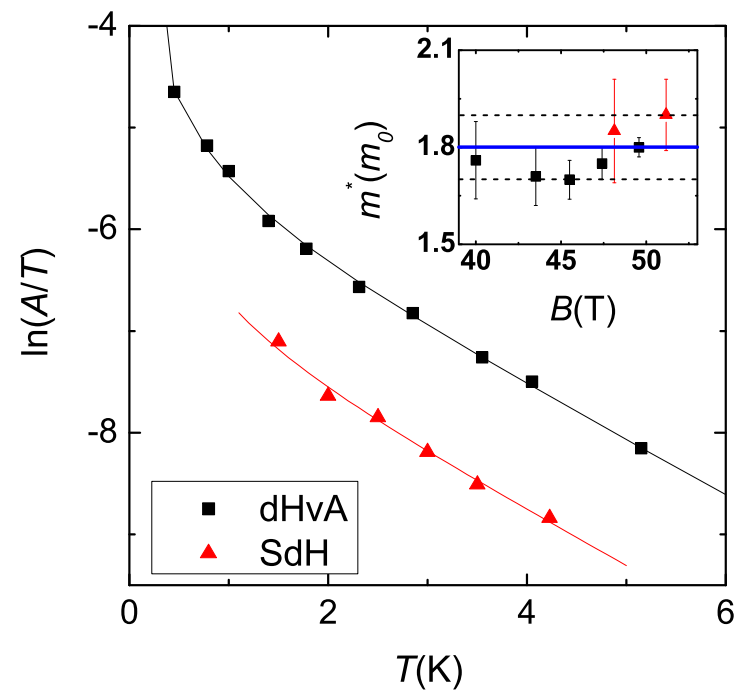

FIG. 2: Temperature dependence of the amplitude of the oscillations $\mathrm{A}$ of the $\mathrm{dHvA}$ and the $\mathrm{SdH}$ oscillations at a mean magnetic field of $47.4 \mathrm{~T}$ and $51.7 \mathrm{~T}$, respectively. Solid lines are best fit to eq. 1 Inset: Field dependence of the effective mass.

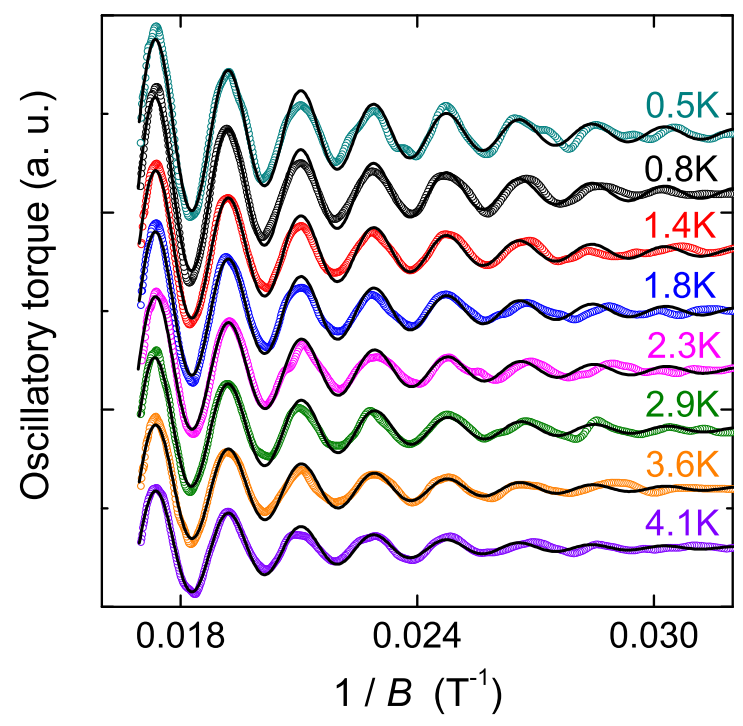

FIG. 3: Oscillatory part of the torque as a function of the inverse field for various temperatures. Solids lines are best fit to eq. 1 with $m^{*}=1.76 m_{0}$ and $T_{D}=6.2 \mathrm{~K}$.

Within our experimental resolution, we can conclude that the effective mass is field-independent, as expected in the standard LK theory [14].

The field dependence of the amplitude of the oscillations leads to a Dingle temperature $T_{D}=6.2 \pm 1.2 \mathrm{~K}$ for dHvA data and converts to a mean free path of $l \sim 160 \AA$ and $w_{c} \tau=0.7 \pm 0.2$ at $35 \mathrm{~T}$. Within our experimental resolution, it is not possible to resolve an extra attenuation corresponding to the effect of superconductivity on the dHvA amplitude. Note that the field range in which $R_{x y}$ 


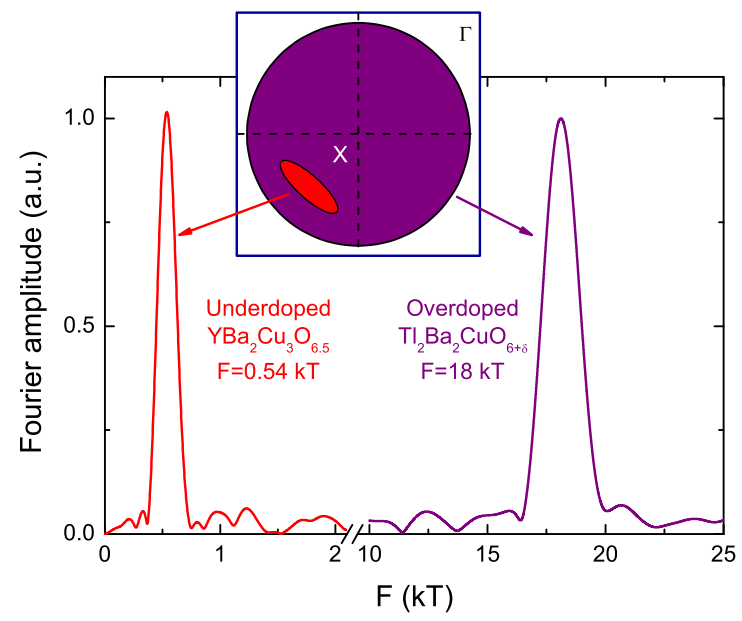

FIG. 4: Fourier transform of the oscillatory part of the torque for underdoped $\mathrm{YBa}_{2} \mathrm{Cu}_{3} \mathrm{O}_{6.51}$ (red) and for overdoped Tl2201 (purple). Insert: Sketch of the FS area deduced from the frequencies of quantum oscillations via the Onsager relation. Note that the location in k-space and the number of small red pocket is at present unknown.

oscillations are detected is too small to derive a reliable Dingle temperature.

Fig. 3 displays the oscillatory torque versus $1 / B$ between $T=4.1 \mathrm{~K}$ and $T=0.5 \mathrm{~K}$. Black solid lines are fits to Eq. 10btained by setting $m^{*}=1.76 m_{0}$ and $T_{D}=$ $6.2 \mathrm{~K}$ for all temperatures. The deduced oscillation frequency and phase factor are $F=540 \pm 4 \mathrm{~T}$ and $\gamma=0.15$ \pm 0.05 , respectively. This fitting procedure confirms that the LK theory, which describes the dHvA oscillations for a generalized 2D Fermi liquid, is appropriate.

The angular dependence of the SdH frequency [9] indicates that QO arise from a quasi-2D FS. The Onsager relation $F=\left(\phi_{0} / 2 \pi^{2}\right) A_{k}$ ( $\phi_{0}$ is the flux quantum) yields a cross sectional area $A_{k}=5.1 \mathrm{~nm}^{-2}$ which corresponds to a small pocket covering $\sim 1.9 \%$ of the first Brillouin zone (FBZ). In overdoped $\mathrm{Tl}_{2} \mathrm{Ba}_{2} \mathrm{CuO}_{6+\delta}$ (Tl2201) superconductor, AMRO [16], ARPES [17] and more recently QO [18] measurements indicate that the FS consists of a quasi-2D hole cylinder of $\sim 65 \%$ of the FBZ area, in agreement with LDA calculation [19]. The sharp contrast between the size of the FS on opposite sides of the phase diagram is illustrated in Fig. 4. The main panel displays the Fourier transform of QO in underdoped $\mathrm{YBa}_{2} \mathrm{Cu}_{3} \mathrm{O}_{6.51}$ (red) and of overdoped Tl2201 (purple) [18]. The inset shows the area of the corresponding orbit in the FBZ. This drastic dissimilarity of FS topology simply reflects the difference in carrier density on opposite sides of the phase diagram. While the large cylinder in the overdoped side corresponds to a carrier density of $1+p$, as predicted by band structure calculations, the band picture fails in the underdoped side, where the carrier number scales more closely with $p$. Other indications pointing to a low carrier concentration in underdoped cuprates come from thermodynamic [20], transport 21] and superfluid density 22].

To be more precise, the carrier density is given by the Luttinger theorem, $n=A_{k} /\left(2 \pi^{2}\right)=F / \phi_{0}$ for each pocket. The frequency $F=530 \mathrm{~T}$ converts to a carrier density of 0.038 carriers per planar $\mathrm{Cu}$ atom. Independently of whether there are 2 or 4 pockets, it gives a number of carriers which is not in agreement with the doping level (10\%). However, a scenario based on a reconstruction of the FS can explain both the negative Hall effect (electron pocket) in the normal state [15] and the apparent violation of the Luttinger sum rule. It assumes that the frequency observed with $\mathrm{SdH}$ and $\mathrm{dHvA}$ effects corresponds to an electron pocket, whose mobility is much higher than that of a larger hole pocket. Note that this larger pocket is not detected in the present measurement, but may have been detected in a recent dHvA study in the same sample [10]. The origin of such reconstruction is still under debate: a reconstruction could occur due to a competing order parameter in the pseudogap phase, e.g. antiferromagnetism [23], d-DW [24, 25] or orbital currents [26], or a stripe-like order could appear close to the $1 / 8$ doping [27].

In summary, we have measured the $\mathrm{SdH}$ and $\mathrm{dHvA}$ effects in underdoped $\mathrm{YBa}_{2} \mathrm{Cu}_{3} \mathrm{O}_{6.51}$ in pulsed magnetic fields up to $60 \mathrm{~T}$. A single frequency $F=530 \pm 10 \mathrm{~T}$ has been reported in both measurements. The data are well described by the LK theory, which accounts for the dHvA oscillations of generalized 2D Fermi liquid. The low frequency corresponds to a small pocket covering $\sim 1.9 \%$ of the FBZ, in sharp contrast with the large cylinder predicted by band structure calculations and observed experimentally in overdoped $\mathrm{Tl}_{2} \mathrm{Ba}_{2} \mathrm{CuO}_{6+\delta}$. The fundamental question is: What causes the FS to undergo such a radical change between the overdoped and the underdoped regions of the phase diagram?

* Electronic address: proust@lncmp.org

[1] N. Doiron-Leyraud et al., Nature 447, 565 (2007).

[2] M.R. Norman et al., Nature 392, 157 (1998).

[3] S. Chakravarty et al., Phys. Rev. B 68, 100504(R) (2003).

[4] N. Harrison et al., Phys. Rev. Lett. 99, 206406 (2007).

[5] M. A. Hossain et al., Nat. Phys. 4, 527 (2008).

[6] A.F. Bangura et al., Phys. Rev. Lett. 100, 047004 (2008).

[7] E.A. Yelland et al., Phys. Rev. Lett. 100, 047003 (2008).

[8] A. Carrington and E.A. Yelland, Phys. Rev. B 76, 140508 (2007).

[9] C. Jaudet et al., Phys. Rev. Lett. 100, 187005 (2008).

[10] S.E. Sebastian et al., Nature 454, 200 (2008).

[11] E. Ohmichi and T. Osada, Rev. Sci. Instrum. 73, 3022 (2002).

[12] R. Liang et al., Physica C 336, 57 (2000).

[13] R. Liang et al., Phys. Rev. B 73, 180505 (2006).

[14] D. Shoenberg, Magnetic Oscillations in Metals (Cambridge University Press, Cambridge, 1984). 
[15] D. LeBoeuf et al., Nature 450, 533 (2007).

[16] N. E. Hussey et al., Nature 425, 814 (2003).

[17] M. Platé et al., Phys. Rev. Lett. 95, 077001 (2005).

[18] B. Vignolle et al., Nature 455, 952 (2008).

[19] D.J. Singh and W.E. Pickett, Physica C 203, 193 (1992).

[20] T. Timusk and B. Statt, Rep. Prog. Phys. 62, 61-122 (2000).

[21] N.E. Hussey, J. Phys. - Condens. Matt., 20, 123201 (2008).
[22] D.M. Broun et al., Phys. Rev. Lett. 99, 237003 (2007).

[23] W.Q. Chen et al., EuroPhys. Lett. 82, 17004 (2008).

[24] I. Dimov et al., Phys. Rev. B 78, 134529 (2008).

[25] D. Podolsky and H.Y. Kee, Phys. Rev. B 78, 224516 (2008).

[26] L. Zhu et al., Phys. Rev. Lett. 100, 057001 (2008).

[27] A.J. Millis and M. Norman, Phys. Rev. B 76, 220503(R) (2007). 